\title{
Spectral Properties of Schrödinger Operators on Decorated Graphs
}

\author{
J. Brüning, V. A. Geiler, and I. S. Lobanov
}

Received November 27, 2004

\begin{abstract}
KEY WORDS: Schrödinger operator, spectral properties, nanostructure, periodic decorated graph,
\end{abstract} quantum network, metric graph, gauge periodic operator.

In the last few years, the spectral theory of the Laplace-Beltrami operator on periodic manifolds has attracted special attention (see, e.g., [1] and the references therein). Not the last of the reasons is the interest in the transport properties of carbon nanostructures composed of fullerenes and nanotubes [2], whose geometry is adequately modeled by the so-called hybrid manifolds, i.e., by topological spaces composed of manifolds of different dimensions. In this paper, we present several results concerning the spectrum of the Schrödinger operator on periodic decorated graphs, which are a special case of periodic hybrid manifolds and, in turn, include metric graphs (quantum networks) [3] as a special case.

In what follows, $\Gamma$ denotes a certain oriented graph with the set $V$ of vertices and the set $E$ of edges. For each $v \in V$, we choose a compact Riemannian manifold $M_{v}$ whose dimension does not exceed 3 , and for each $e \in E$, we choose a segment

$$
M_{e}=\left[a_{1}(e), a_{2}(e)\right] \subset \mathbb{R}, \quad \text { where } \quad a_{1}(e) \leq a_{2}(e)
$$

A topological space obtained from the disjoint union $Y$ of manifolds $M_{v}$ and segments $M_{e}$ by gluing each point $a_{1}(e)$ (respectively, $a_{2}(e)$ ) to the manifold $M_{v}$, where $v$ is the starting point (respectively, the endpoint) of the edge $e$, will be called a decorated graph $X$. We note that if all the manifolds $M_{v}, \quad v \in V$, are zero-dimensional and all the segments $M_{e}, e \in E$, are nondegenerate, then the decorated graph is exactly a metric graph.

By $\Xi$ we denote the disjoint union of the sets $V$ and $E$. Then, under the natural definition of the space $L^{2}(X)$, we have the identification

$$
L^{2}(X) \simeq \bigoplus_{\xi \in \Xi} L^{2}\left(M_{\xi}\right)
$$

For each $\xi \in \Xi$, we choose a Schrödinger operator $H_{\xi}$ in $L^{2}\left(M_{\xi}\right)$ determined by a second-order differential expression on $M_{\xi}$ with sufficiently smooth coefficients and (for $\xi \in E$ ) by self-adjoint boundary conditions at the endpoints of the segment $M_{\xi}$. Then the Schrödinger operator on $X$ can be defined as follows. Suppose that $S_{\xi}$ is the restriction of $H_{\xi}$ to the set of all functions defined on the domain $\mathcal{D}\left(H_{\xi}\right)$ and vanishing at the points of gluing, $S$ is the direct sum of all $S_{\xi}$, and $\xi \in \Xi$. Any self-adjoint extension of the operator $S$ will be called a Schrödinger operator on $X$. It is convenient to describe all the Schrödinger operators generated by $S$ by using the Krein formula for resolvents. To do this, we denote the direct sum of all $H_{\xi}$ by $H^{0}$ and, for each $\xi \in \Xi$, introduce an auxiliary Hilbert space $\mathcal{G}_{\xi}$ whose dimension coincides with the deficiency number of 
the operator $S_{\xi}$. Suppose that $\gamma_{\xi}$ and $Q_{\xi}$ are, respectively, the $\Gamma$-field and the $\mathcal{Q}$-function of the pair $\left(H_{\xi}, S_{\xi}\right)$ [4]; in particular, $\gamma_{\xi}(z)$ (respectively, $Q_{\xi}(z)$ ) is a bounded linear operator from $\mathcal{G}_{\xi}$ to $L^{2}\left(M_{\xi}\right)$ (respectively, from $\mathcal{G}_{\xi}$ to $\mathcal{G}_{\xi}$ ) for each regular value $z$ of the operator $H^{0}$. Now, we set

$$
\mathcal{G}=\bigoplus_{\xi \in \Xi} \mathcal{G}_{\xi}, \quad \gamma=\bigoplus_{\xi \in \Xi} \gamma_{\xi}, \quad Q=\bigoplus_{\xi \in \Xi} Q_{\xi} .
$$

Then the following formula determines a bijection between the resolvents of all self-adjoint extensions $H$ of the operator $S$ and the self-adjoint (generally, multivalued) operators $\Lambda$ in $\mathcal{G}$ :

$$
(H-z)^{-1}=\left(H^{0}-z\right)^{-1}-\gamma(z)[Q(z)-\Lambda]^{-1} \gamma^{*}(\bar{z}) .
$$

The extension of $H$ corresponding to $\Lambda$ will be denoted by $H_{\Lambda}$. An operator $\Lambda$ is single-valued if and only if $H_{\Lambda}$ and $H^{0}$ are disjoint, i.e.,

$$
\mathcal{D}\left(H_{\Lambda}\right) \cap \mathcal{D}\left(H^{0}\right)=\mathcal{D}(S) .
$$

To simplify the subsequent statements, we assume that $H_{\Lambda}$ and $H^{0}$ are disjoint. We note that, in fact, this assumption does not lead to a loss of generality: the graphs of all $\Lambda$ are exactly Lagrangian subspaces in $\mathcal{G} \times \mathcal{G}$ with respect to the standard skew-Hermitian product [5]; obviously, the graphs of single-valued operators form Lagrangian planes in general position.

We shall consider periodic decorated graphs and gauge-periodic operators $H_{\Lambda}$ on them. To do this, we assume that a discrete group $G$ acts freely on a graph $\Gamma$; in particular, the action of the group $G$ on $\Xi$ is given. Assuming that $M_{g \xi}=M_{\xi}, g \in G, \xi \in \Xi$, and the points of gluing are invariant under the action of $G$ on $Y$, we obtain the natural action of $G$ on $X$. Now, let $\sigma$ be a 2-cocycle on $G$ ranging in $\mathbf{U}(1)$. Closed linear operators $A$ in $L^{2}(X)$ with $(G, \bar{\sigma})$-invariant resolvents are said to be gauge-periodic. If we have $\sigma=1$ in this case, then $A$ is said to be periodic. In what follows, we assume that $H_{g \xi}=H_{\xi}$ for any $g \in G$. Then we can easily prove that the Schrödinger operator $H_{\Lambda}$ is gauge-periodic if and only if $\Lambda$ is $(G, \bar{\sigma})$-invariant. Everywhere below, the last condition is assumed to be satisfied.

Next, we assume that $G$ acts on $\Gamma$ with finitely many orbits. We let $F$ denote the fundamental set of the action of $G$ on $\Xi$, i.e., a subset $\Xi$ containing a single representative of each orbit. By $C_{r}^{*}(G, \sigma)$ we denote the reduced twisted group $C^{*}$-algebra of the group $G$ with factor $\sigma[6]$, and by $\mathcal{H}_{f}$ (respectively, by $\mathcal{G}_{f}$ ) the space $\bigoplus_{\xi \in F} L^{2}\left(M_{\xi}\right)$ (respectively, $\bigoplus_{\xi \in F} \mathcal{G}_{\xi}$ ). The space of all compact linear operators in the Hilbert space $\mathcal{H}$ will be denoted by $\mathcal{K}(\mathcal{H})$, and the standard trace in

$$
C_{r}^{*}(G, \sigma) \otimes \mathcal{K}\left(\mathcal{H}_{f}\right)
$$

will be denoted by $\operatorname{Tr}$. Since $\mathcal{G}_{f}$ is a finite-dimensional space, the following theorem significantly simplifies the study of the spectrum of the gauge-periodic operator $H_{\Lambda}$ (in this theorem and in what follows, we identify the spaces $L^{2}(X)$ and $\mathcal{G}$ with $l^{2}\left(G, \mathcal{H}_{f}\right)$ and $l^{2}\left(G, \mathcal{G}_{f}\right)$, respectively).

Theorem 1. Let $\Lambda$ belong to the $C^{*}$-algebra $C_{r}^{*}(G, \sigma) \otimes \mathcal{K}\left(\mathcal{G}_{f}\right)$. Then the resolvent of the operator $H_{\Lambda}$ belongs to $C_{r}^{*}(G, \sigma) \otimes \mathcal{K}\left(\mathcal{H}_{f}\right)$.

The assumption of Theorem 1 is satisfied for the very important case in which $\Lambda$ is a bounded operator in $\mathcal{G}$ and its standard matrix representation has only finitely many nonzero diagonals. Moreover, we assume that $G$ is equipped with a left-invariant metric $\rho$. Then, using the methods proposed in [7], we can prove the following theorem.

Theorem 2. Suppose that $\Lambda$ is a bounded operator and there exist constants $C$ and $\delta>0$ such that, for all $\alpha, \beta \in G$, the matrix elements $\Lambda(\alpha, \beta)$ satisfy the condition

$$
\|\Lambda(\alpha, \beta)\| \leq C \eta(\rho(\alpha, \beta))^{-1-\delta},
$$


where $\eta(R)$ is the number of elements in the ball of radius $R$. Then $\Lambda \in C_{r}^{*}(G, \sigma) \otimes \mathcal{K}\left(\mathcal{G}_{f}\right)$.

In what follows, we assume that $H_{\Lambda}$ is semibounded below, which is the case under very general assumptions on $H_{\xi}$ and $\Lambda$. We fix a real number $E^{\prime}<\inf \operatorname{spec}\left(H^{\Lambda}\right)$ and, for $E \notin \operatorname{spec}\left(H^{\Lambda}\right)$, define

$$
N(E):= \begin{cases}\operatorname{Tr} P_{\left[E^{\prime}, E\right]}, & E \geq E^{\prime} \\ 0, & E<E^{\prime}\end{cases}
$$

where $P$ is the spectral projection operator for $H_{\Lambda}$.

Corollary 1. Under the assumptions of Theorem 1, the values of $N(E)$ lie in a countable set of real numbers $\operatorname{Tr}\left(K_{0} C_{r}^{*}(G, \sigma)\right)$, where $K_{0}$ is the corresponding component of the $K$-functor on the category of $C^{*}$-algebras.

It is said that the pair $(G, \sigma)$ has the Kadison property if the traces of all nonzero self-adjoint projection operators from $C_{r}^{*}(G, \sigma) \otimes \mathcal{K}\left(l^{2}\right)$ are bounded away from zero. In particular, each pair $\left(\mathbb{Z}^{m}, 1\right), m \geq 1$, has the Kadison property; more examples can be found in [8].

Corollary 2. If, under the assumptions of Theorem 1, the pair $(G, \sigma)$ has the Kadison property, then the spectrum of the operator $H_{\Lambda}$ has a zone structure.

It is said that the $C^{*}$-algebra $A$ has the $R R I_{0}$ property if $A$ has an exact state $F$ such that each self-adjoint element from $A$ can be approximated (with arbitrary accuracy) by an element with finite spectrum whose spectral projection operators are arbitrarily small on $F$ [9]. The proof of the following corollary is based on an argument from [10].

Corollary 3. Suppose that the assumptions of Theorem 1 are satisfied. Suppose additionally that the $C^{*}$-algebra $C_{r}^{*}(G, \sigma) \otimes \mathcal{K}\left(\mathcal{H}_{f}\right)$ has the $R R I_{0}$ property. Then the operator $H_{\Lambda}$ can be approximated with arbitrary accuracy in the sense of uniform resolvent convergence by operators with a Cantor spectrum from $C_{r}^{*}(G, \sigma) \otimes \mathcal{K}\left(\mathcal{H}_{f}\right)$.

Next, we assume that $G$ is an Abelian group without torsion; then $G \simeq \mathbb{Z}^{d}$, and, in $G$, we fix the standard metric $\rho$ of the group $\mathbb{Z}^{d}$.

Theorem 3. Suppose that

$$
\Lambda(\alpha, \beta) \leq c_{1} \exp \left(-c_{2} \rho(\alpha, \beta)\right)
$$

with constants $c_{1}, c_{2}>0$. Then $H_{\Lambda}$ has a zone spectrum without any singular continuous component.

Remark. Simple examples show that, under the assumptions of Theorem 3, the operator $H_{\Lambda}$ can have eigenvalues.

In [11], one can find examples of the Schrödinger operator on a periodic decorated graph $X$, $\operatorname{dim} X \geq 2$, whose spectrum has infinitely many gaps, i.e., for which the Bethe-Sommerfeld hypothesis is not true [12]. We show that this hypothesis is not true for a rather wide class of periodic decorated graphs.

Theorem 4. Suppose that $G$ acts transitively on the set of vertices of the graph $\Gamma$. Suppose that the operator $H_{v}$ (which is independent of $v$ in this case) has infinitely many eigenvalues whose multiplicity is greater than the degree of the vertex $v$ (which is also independent of $v$ ). Suppose also that all segments $M_{e}$ are degenerate. Then, when the points of gluing are in general position, the spectrum of $H_{\Lambda}$ has infinitely many lacunas both in the sense of the Lebesgue-Riemann measure and in the sense of the Baire category. 


\section{ACKNOWLEDGMENTS}

This research was supported by the Russian Foundation for Basic Research under grant no. 0201-00804, by INTAS, and by DFG.

\section{REFERENCES}

1. O. Post, J. Differential Equations, 187 (2003), 23-45.

2. H. Aoki et al., Phys. Rev. B, 65 (2002), 035102.1-035102.8.

3. P. Kuchment, Waves in Random Media, 14 (2004), 107-128.

4. M. G. Krein and G. K. Langer, Funktsional. Anal. i Prilozhen. [Functional Anal. Appl.], 5 (1971), no. 2, 59-71.

5. B. S. Pavlov, Uspekhi Mat. Nauk [Russian Math. Surveys], 42 (1987), no. 6, 99-131.

6. L. Auslander and C. C. Moore, Mem. Amer. Math. Soc. (1966), no. 62, 1-199.

7. J. Brüning and T. Sunada, Astérisque, 210 (1992), 65-74.

8. V. Mathai, Tôhoku Math. Publ., 20 (2001), 107-124.

9. M. Choi, G. Elliott, and N. Yui, Invent. Math., 99 (1990), 225-246.

10. M. J. Gruber, J. Math. Phys., 42 (2001), 2438-2465.

11. J. Brüning, P. Exner, and V. A. Geyler, J. Phys. A. Math. Gen., 36 (2003), 4875-4890.

12. M. M. Skriganov, Trudy Mat. Inst. Steklov [Proc. Steklov Inst. Math.], 171 (1985), 1-124.

(J. Brüning, I. S. Lobanov) Institut für Mathematik DER Humboldt-Universität ZU Berlin

E-mail: (J. Brüning) bruening@mathematik.hu-berlin.de

(I. S. Lobanov) lobanov@math.hu-berlin.de

(V. A. Geiler, I. S. Lobanov) N. P. Ogarev Mordovian State University

E-mail: (V. A. Geiler) geyler@mail.ru, geyler@mrsu.ru

(I. S. Lobanov) lobanovis@math.mrsu.ru 